\title{
La educación temprana para el desarrollo cognitivo en niños de contextos carcelarios
}

\author{
Early education for cognitive development in children from prison contexts
}

Educação infantil para o desenvolvimento cognitivo em crianças em contextos de prisão

Fernando Eli Ledesma Pérez

fledesma25@gmail.com

https://orcid.org/0000-0003-4572-1381

\author{
Sixto Vega Vilca \\ cvegacs@ucv.edu.pe \\ https://orcid.org/0000-0002-2755-8819
}

\section{Juana María Cruz Montero \\ jcruzmo@ucv.edu.pe \\ https://orcid.org/0000-0002-7772-6681}

Silvia Vanessa Rodríguez Melgar
srodriguezm1@ucv.edu.pe

Grupo de investigación: investigación cultura y desarrollo, Universidad César Vallejo Lima, Perú

\section{RESUMEN}

La educación temprana es una tarea desarrollada por personas que rodean al infante desde su nacimiento, consiste en el acompañamiento en su proceso de desarrollo: biológico, afectivo y social; es prioritaria en los tres primeros años en los que aprende a caminar, hablar y a relacionarse con otros. El objetivo fue determinar la influencia de la aplicación de un programa de educación temprana en el desarrollo cognitivo en infantes menores de tres años en un establecimiento penitenciario de mujeres; investigación de tipo básica, nivel explicativo, diseño pre-experimental, con un grupo de 40 niños. Los resultados demostraron que se trabajó desde la propuesta teórica de Erikson (1998), participaron cuatro docentes universitarios y cinco estudiantes del último ciclo de educación inicial, se aplicó un programa en dos semestres y se concluyó que la aplicación de un programa educación temprana influyó de manera significativa en el desarrollo cognitivo de los niños y en otras áreas del desarrollo.

Palabras clave: Educación inicial; Desarrollo cognitivo; Confianza; Autonomía; Vergüenza/culpa

\section{ABSTRACT}

Early education is a task developed by people who surround the infant from birth, it consists of accompanying him/her in his/her development process: biological, affective and social; it is a priority in the first three years in which they learn to walk, talk, relate to others and control their physiological needs; the research sought to determine the influence of the application of an early education program in the cognitive development of infants under three years of age in a women's penitentiary establishment; It was a basic research, explanatory level, pre-experimental design, with a group of 40 children. The results show working from Erikson's theoretical proposal (1998), with the participation of four university teachers and five students of the last cycle of early education, a program was applied in two semesters and it was concluded that the application of an early education program had a significant influence on the cognitive development of children and in other areas of development.

Key words: Initial education; Cognitive development; Confidence; Autonomy; Shame

\section{RESUMO}

A educação precoce é uma tarefa realizada por pessoas que rodeiam o bebé desde o nascimento, e consiste em acompanhálos no seu processo de desenvolvimento: Biológico, afectivo e social; é uma prioridade nos primeiros três anos em que aprendem a andar, falar, relacionar-se com os outros e controlar as suas necessidades fisiológicas; a investigação procurou determinar a influência da aplicação de um programa de educação precoce no desenvolvimento cognitivo de bebés com menos de três anos de idade num estabelecimento penitenciário feminino; Tratava-se de uma investigação básica, a nível explicativo, concepção préexperimental, com um grupo de 40 crianças. Os resultados mostram trabalhando a partir da proposta teórica de Erikson (1998), com a participação de quatro professores universitários e cinco estudantes do último ciclo de educação precoce, um programa foi aplicado em dois semestres e concluiu-se que a aplicação de um programa de educação precoce teve uma influência significativa no desenvolvimento cognitivo das crianças e em outras áreas de desenvolvimento.

Palavras-chave: Educação inicial; Desenvolvimento cognitivo; Confiança; Autonomia; Vergonha 


\section{INTRODUCCIÓN}

La vida en las cárceles está asociada al hacinamiento, transgresión y violencia, en ese sentido, este escenario no es el ideal para la permanencia de infantes. En Perú, el Artículo 12 del Reglamento del Código de Ejecución Penal (2013) establece que las reclusas con sentencia firme y consentida tienen el derecho de permanecer con sus hijos hasta que estos alcancen los tres años de edad, después de este período los niños serán entregados a la persona correspondiente de acuerdo con las regulaciones al respecto, o en su defecto, procederá la aplicación de la colocación familiar o su derivación a una institución de tutela conforme a lo dispuesto en el Código del niño y del adolescente (artículo 12). Por lo tanto, conforme a la legislación peruana, los infantes pueden permanecer con sus madres hasta cumplir los tres años.

La comisión de la Mujer y familia, Período 2011-2012, emitió informe en el que precisaba que según registros oficiales del Instituto Nacional Penitenciario (2012) la población carcelaria hasta marzo 2012, fue del $6.2 \%$ mujeres y $93.8 \%$ hombres. Así mismo señalo que la población penitenciaria femenina en un $38 \%$ se encontraba con sentencia y un $62 \%$ están en calidad de procesadas. Como puede verse, las reclusas representan una población baja respecto a los varones y la mayoría de ellas estaba sin sentencia.

La población carcelaria se incrementa cada año, lo cual ha generado una disminución en los servicios que se les ofrece a quienes conviven con sus hijos, esto afecta: alimentación, buen trato y aseguramiento de una estancia cómoda en la cuna-jardín; como consecuencia se agudiza el hacinamiento porque la infraestructura ha excedido su capacidad de albergue, esta situación repercute en forma directa en los niños, perjudica su desarrollo cognitivo y podrían generar otras deficiencias especialmente en su desarrollo emocional y social. Entonces, el incremento de la población de reclusas e infantes sin asignarse nuevos espacios físicos, afecta los servicios que reciben, dentro de ellos el servicio educativo en la cuna jardín.

Los infantes menores de tres años han sido mencionados como niños invisibles en el informe elaborado por el Instituto Nacional de las Mujeres (2002), se precisa que son las víctimas invisibles del crimen y del sistema penitenciario; quienes nacieron en el penal no hicieron nada malo, ni cometieron algún delito, no obstante, soportan el estigma del crimen; su derecho a una crianza normal se ve afectada por la acción sus padres (Aguilera, 2011; y Robertson, 2012). La Organización de las Naciones Unidas-ONU (2005) citada por Briceño (2006) refiere que estos infantes se encuentran expuestos a condiciones de alta vulnerabilidad, dadas las características del contexto carcelario y penitenciario, ellos ven amenazados sus derechos, en especial, a la salud, la integridad, la calidad de vida, la dignidad, el amparo y el desarrollo integral. En este sentido, los organismos internacionales han considerado este fenómeno como perjudicial.

Frente a la situación de infantes menores de tres años institucionalizados en una cárcel de mujeres y ante el innegable valor que representa su permanencia para la preservación del contacto entre madre e hijo, la construcción del apego y las estructuras psicológicas de seguridad, confianza y protección; es necesario el abordaje de los efectos de la interrupción del vínculo familiar (nuclear y extenso) el cual afecta su mundo en construcción y mella la imagen materna que percibe, por lo que desde lo académico-formativo de la universidad debe paliarse su afectación cognitiva, emocional, social y espiritual a través de programas que reduzcan su vulnerabilidad y eviten el sufrimiento de daños o injusticias. 
Antony (2007) precisó que la cárcel es un espacio en el que el infante se relaciona y aprende de manera negativa, porque la mayoría de acciones ocurren en un contexto de violencia; Kalinsky (2013) sostuvo que los gritos, los diversos pleitos, los golpes y la violencia que es común en el ambiente carcelario son perjudiciales para los infantes y generan dificultades en sus relaciones interpersonales. Pese a la existencia de posibilidades que el infante tenga un buen desarrollo, la falta de lugares libres y convenientes es una limitación, Jiménez (2002) aduce que en la penitenciaria la estimulación es extraordinariamente baja, explicable por prácticas sociales incoherentes, por el desplazamiento, los materiales, los juguetes y la variedad de las actividades diarias, las que inciden en el desarrollo educativo deficitario o distorsionado.

Las reclusas madres refieren que sus hijos son atendidos por el programa de control y crecimiento, que reciben evaluaciones periódicas para ver su desarrollo físico y psicomotor; ellas reciben orientación sobre alimentación y estimulación temprana. Sin embargo, carecen de los materiales para leerles cuentos, carecen de espacios para jugar con sus hijos y de orientaciones teóricas para la detección de problemas en sus hijos. Cabe precisar que no hay seguimiento al desarrollo cognitivo en los procesos de percepción, memoria, pensamiento y lenguaje. Por ello, el objetivo de esta investigación fue determinar la influencia de la aplicación de un programa de educación temprana en el desarrollo cognitivo en niños menores de tres años en un establecimiento penitenciario de mujeres.

Investigaciones sobre el tema y que se tomaron como antecedentes, ayudan a la comprensión del problema, así se tiene que Méndez (2019) quien investigó en una prisión femenina de Lima, caracterizó a la cárcel por la rudeza en el trato, pensada para ejercer sanciones y en cuya concepción no se incluyó la posibilidad de acoger infantes, por lo que se hicieron adaptaciones para albergarlos; ello genera contrastes en la convivencia por compartir las mismas condiciones de encierro, las redes familiares son un soporte para los cuidados y la provisión, agregó que el encierro no se agota con la privación de la libertad, sino que impacta al entorno fuera de la prisión y la presencia de los infantes son evidencia de ello. Dorigo y Janampa (2012) investigaron la brecha entre la normativa y la situación penitenciaria de la gestante y madre con hijos, concluyeron que no existe servicio de psicología que las apoye y guíe, falta servicio gíneco-obstétrico y pediátrico permanente, falta acceso a talleres educativos y laborales, y apoyo para la comercialización de los productos hechos en el penal, recomendaron mejorar la alimentación, organización de una Escuela de Madres para el cuidado de los niños, un espacio exclusivo para favorecer el vínculo materno filial y evitar la desestructuración familiar.

Montalvo (2007) investigó el ejercicio político de la maternidad en la prisión de mujeres del Ecuador, en la que la maternidad entendida y pensada por las madres en prisión, desde una mirada que no investiga delincuentes o criminales, sino a la maternidad como una forma de relación respecto a la vida cotidiana y encontró evidencias que viven su maternidad de manera compartida y colectiva y concluyó que se debe hacer un mayor esfuerzo para favorecer el vínculo materno-filial y familiar. Amaya et al. (2008) investigaron las reacciones emocionales, cognitivas y de comportamiento experimentadas por un conjunto de madres privadas de su libertad en una prisión de Costa Rica y concluyeron que cuando los separan madres e hijos luego de su estancia en prisión se ofrecen recomendaciones y pautas para que las madres estén preparadas para la separación de sus hijos y para que ellos tengan una buena niñez y logren una buena socialización. 
Manríquez (2016) investigó en Chile, los programas de cuidado de hijos e hijas de mujeres privadas de libertad, además investigó las percepciones, opiniones y evaluaciones de las madres involucradas en el sistema con relación a las experiencias de cuidado pequeño y concluyó que los niños son un estímulo para las madres que están en prisión y cambian su perspectiva de la vida, queriendo sobresalir y obtener una mejor vida para su familia. Becerra (2004) investigó en Colombia sobre las visitas de niños a sus padres en contexto penitenciario para prevenir el maltrato infantil y fortalecer el vínculo familiar, concluyó que los hijos son el motor de vida del interno, la crianza debe ser con base en disciplina con amor; es necesario optimizar en calidad, la cantidad del tiempo compartido en la visita.

Merino (2015) realizó una investigación en España para conocer la situación del colectivo carcelario que tenían niños bajo su cuidado tanto dentro como fuera de la prisión, prestando especial atención a la educación que reciben, concluyó que la permanencia de prisión es difícil, mucho más para las madres, imposibilita desarrollar la maternidad, a pesar de las leyes que hacen creer que es posible; la infraestructura es deficitaria, los módulos adaptados se han reformado, pero no dejan de tener esa esencia de prisión que no permite que las madres sean tales; incluso si los barrotes de una prisión están pintados, sigue siendo una prisión. Gasser (2016) investigó en Bolivia sobre los procesos de socialización, referentes y modelos sociales en niños que viven en las cárceles bolivianas, con una muestra de 50 niños con edades entre 6 y 12 años y con cuarenta madres de familiares detenidos por asesinato, robo, drogas $\mathrm{y}$ algunos con agravantes; los resultados mostraron que el encarcelamiento tiene como consecuencia la desesperanza asimilada, especialmente para aquellos que han estado encarcelados durante algunos años, ya que la incertidumbre de su situación legal les impide edificar un proyecto de vida e imaginar un futuro para ellas y sus hijos.

Los antecedentes citados evidencian los diferentes puntos de vista y resultados sobre la vida de las madres en prisión, quienes asumen diversos roles y actuaciones, destacando como situaciones especialmente críticas la maternidad y la permanencia de sus hijos en los recintos carcelarios, pese a las implicancias que esta condición tiene para ellos, sin embargo, tienen efectos favorables en la vida de sus madres quienes se sienten acompañadas $y$ creen que en esa circunstancias pueden inculcarles valores.

Desde la teoría, se han referido a la educación temprana Ramírez-Abrahams et al. (2014) quienes se refieren a este constructo como aquel destinado a la satisfacción de las necesidades básicas y los servicios de calidad en educación. Chokler (1988) consideró que la atención temprana hace referencia al cuidado que se prodiga al niño desde su nacimiento, es una actividad de carácter social y de nivel profesional; se fundamenta en la concepción del desarrollo que considera el rol del adulto, familia, comunidad y docentes, en el apoyo y la sustentación del desarrollo del infante; por lo tanto, esta concepción engloba a todas las personas e instituciones que tienen responsabilidades con la infancia. Moreno (2012) consideró que, en la relación de una persona mayor con el infante, son esenciales la seguridad afectiva que genera una eficacia del vínculo y de enlace y se consigue con interacciones permanentes; estas permiten que el psiquismo precario supere las experiencias de impotencia en tanto se satisfacen las necesidades biológica y afectiva que el infante es incapaz de satisfacer por sí mismo. Los principios propuestos por Moreno (2012) fueron desarrollados por Emmi Pikler, quien prestaba especial atención al desarrollo motor espontáneo en la primera 
infancia. Wallon (1976) muestra que la raíz del pensamiento, la relación entre el tono muscular, la emoción, la expresión y la comunicación, entre el tono y el psiquismo son generados en la primera infancia. Bowlby (1988) y Hartmann et al. (1950) citados por Bowly, muestran suficientes referencias desde la teoría psicoanalítica del apego, sobre la importancia del vínculo madre-hijo en los primeros años de vida y de las repercusiones de esta en la vida posterior.

Sobre el desarrollo cognitivo, el teórico fue Erikson (1998) citado por Bordignon (2006) quien admite la influencia de la teoría psicoanalítica de Freud y a partir de su experiencia antropológica, indicó que la presencia del ser humano depende de todas las fases o de sus tres procesos de organización complementarios: (a) El proceso biológico que encierra la organización que se encuentra subordinada de los procedimientos biológicos, orgánicos y el desarrollo fisiológico - el soma; (b) el proceso psíquico que encierra las experiencias individuales en síntesis del 'yo', los procesos psíquicos y la práctica personal y su relación - la psique; y, (c) la parte ética-social: que encierra la organización, costumbres y la parte religiosa de las personas y de la humanidad, expresadas en principios y valores de orden social - el ethos. Erikson (1998) dijo que, en cualquier experiencia clínica, educacional o formativa, estos procesos están integrados, situación que facilita su estudio con diversos métodos, desde las perspectivas de la atención somática, psíquica o social. Erikson (1998) sostuvo que el principio epigenético involucra que cada uno de los seres vivientes tenga un plano básico de desarrollo por fases, y es desde este plano que se agregan las partes, cada uno con su propio tiempo (de aprender) de ascensión, maduración y ejercicio, hasta que todos han surgido para formar un todo en funcionamiento. No se debe dar todo de manera rápida porque se estaría forzando su maduración (p. 123). Erikson describió que, para cada fase o desarrollo, existe una característica central básica y una crisis psicosocial dialéctica básica. Cada una de estas tiene un potencial sintónico característico de ello para destacar. El potencial de su antítesis. En este caso su potencialidad se contrapone dialécticamente, de modo que la resolución de cada crisis da como resultado el surgimiento de fuerza o calidad básica. A su vez, la fuerza simpática también presenta una contradicción antipática que sigue siendo una amenaza constante para el ser humano y para la sociedad o comunidad.

Las dimensiones del desarrollo cognitivo propuestas por Erikson (1998) son (1) Confianza básica/ desconfianza básica, al respecto Boeree (2002) sostuvo que la confianza básica se da por la familiaridad, estabilidad y continuación, allí el infante aprende a tener confianza y domino de su propio cuerpo; si los padres se separan y no satisfacen sus necesidades, el infante desarrollará una desconfianza, Erikson (1998) sostuvo que si se logra a obtener un equilibrio, en el infante desarrollará la virtud de la esperanza, es una creencia en la que se considera que el ser humano desarrolla una posible solución al final del camino no importa que las cosas estén mal. (2) Autonomía, Boeree (2002) sostuvo que si los padres consienten que el infante manipule e investigue su ambiente, desarrollará un sentido de independencia o autonomía, por ello debe permitirse que examine, manipule y explore su ambiente. (3) Vergüenza/culpa, Boeree (2002) sostuvo que cuando no se favorece la autonomía, desarrolla sentido de vergüenza/culpa; la burla a sus esfuerzos puedellevarle a sentirse muy avergonzado, y dudar de sus destrezas. Erikson (1998) añadió que un poco de vergüenza e incertidumbre no solo es inevitable, sino que incluso es algo bueno, sin ella, se desarrollará el arrebato, una especie 
de premeditación sin vergüenza que más tarde, en la infancia tardía o incluso en la edad mayor, se exteriorizará sin tener en cuenta los límites y las injusticias que esto puede producir. Por ello, el objetivo de esta investigación fue determinar la influencia de la aplicación de un programa de educación temprana en el desarrollo cognitivo en niños de contextos carcelarios.

La investigación es importante porque describe la influencia de un programa educativo en el desarrollo cognitivo de la confianza, autonomía y vergüenza/culpa de los infantes que permanecen con sus madres en una prisión, se aproxima a la comprensión de las necesidades y los problemas respecto a su desarrollo integral; es conveniente porque se realizó luego de un diagnóstico que priorizó al niño como sujeto significativo, y luego se implementó un programa de intervención pedagógico para el favorecimiento de su desarrollo social, cognitivo, emocional y espiritual. Su relevancia social radica en que el programa se ejecutó dos semestres dentro de la prisión, resultando beneficiarias indirectas sus madres; con las profesoras se revisó el corpus teórico y se ejecutaron técnicas de atención temprana para el mejoramiento de las condiciones de permanencia del infante en el establecimiento. Se generaron experiencias de aprendizaje en atención temprana y el desarrollo cognitivo desde la propuesta de Erikson. Se pretende aportar al conocimiento temático de niños en contextos carcelarios y con grandes riesgos de vulnerabilidad; así mismo, se persigue generar nuevas líneas de investigación sobre educación en contextos carcelarios.

\section{MÉTODO}

La investigación fue de tipo aplicada, de nivel explicativo el cual consiste en aplicar un programa y ver sus efectos durante un período de tiempo, el programa se realiza para la variable independiente y los efectos se miden en la variable dependiente; diseño experimental de tipo preexperimental, la variable independiente que fue la educación temprana para la cual se diseñó un programa aplicado durante dos semestres académicos, para ver su influencia en la variable dependiente que fue el desarrollo cognitivo. Con respecto al tiempo fue de corte longitudinal porque se trabajó en dos semestres y se realizaron sucesivas mediciones.

El muestreo no probabilístico, de tipo intencional se basa en el juicio personal del investigador o lo que ellos pueden aportar a la investigación y es intencionado o de conveniencia porque la muestra es seleccionada según el criterio y accesibilidad del investigador (Tamayo y Tamayo, 2003).

Fueron incluidos todos los niños que estaban en el Centro Penitenciario, la cifra era variable, siendo el promedio 40. Al inicio hubo 36 niños y llegó a 44, por ello, se eligieron 40 como población muestra. Algunos al cumplir los tres años eran externados. Las madres aceptaron su participación y de manera paralela se hicieron talleres con ellas para mejorar la relación y el cuidado de los niños. Los criterios de eliminación se aplicaban cuando las madres de familia obtenían su libertad o los niños cumplían tres años y eran externados, la cantidad y número de los conformantes de la muestra están en la Tabla 1. 
Tabla 1. Población muestra de infantes.

\begin{tabular}{lccc}
\hline \multicolumn{1}{c}{ Edades } & Frecuencia & Porcentaje & Porcentaje acumulado \\
\hline Lactantes hasta 6 meses & 6 & 15,0 & 15,0 \\
Gateadores. 7 a 12 meses & 12 & 30,0 & 45,0 \\
Caminantes. 13 a 24 meses & 14 & 35,0 & 80,0 \\
Exploradores. Más de 25 meses & 8 & 20,0 & 100,0 \\
Total & $\mathbf{4 0}$ & $\mathbf{1 0 0 , 0}$ & \\
\hline
\end{tabular}

Se empleó la técnica de la observación en cada una de las sesiones, se registraron las conductas de los infantes tanto de confianza/desconfianza, autonomía y vergüenza/culpa, además de las respuestas emocionales sociales que fueron valoradas de forma cualitativa. Se empleó una lista de cotejo de 42 ítems, a la dimensión 1 confianza/ desconfianza se asignó un peso de $21 \%$ (9 ítems) a la dimensión 2 un peso de $29 \%$ (12 ítems) y a la dimensión 3 vergüenza/culpa un peso de 50 \% (21 ítems). La validez de contenido se logró la decisión jueces, sin embargo, también fue sometida a validez de constructo y a validez de consistencia interna, en todos los casos, los resultados fueron favorables. La confiabilidad se calculó con el coeficiente de confiabilidad el Alfa de Cronbach con resultados altos $(, 893)$.
El método de análisis de datos fue cuantitativo, se empleó estadística descriptiva para ver cómo se comportaban los niveles de desarrollo y la estadística inferencial para ver si los cambios que se registraban de manera progresiva eran significativos.

\section{RESULTADOS Y DISCUSIÓN}

Para el desarrollo de la investigación, antes de la aplicación del programa se aplicó la prueba de entrada, también llamada pretest, los resultados para el desarrollo cognitivo de confianza, autonomía y vergüenza/culpa, mostraron que el $55 \%$ de los infantes se ubicó en el nivel bajo, el $25 \%$ en el nivel medio y el $20 \%$ en el alto (Tabla 2 ).

Tabla 2. Resultados descriptivos del pretest.

\begin{tabular}{lcccc}
\hline & Niveles & Frecuencia & Porcentaje & Porcentaje acumulado \\
\hline Bajo & 22 & 55,0 & 55,0 \\
Medio & 10 & 25,0 & 80,0 \\
Alto & 8 & 20,0 & 100,0 \\
Total & $\mathbf{4 0}$ & $\mathbf{1 0 0 , 0}$ & \\
\hline
\end{tabular}


Luego de la aplicación del programa por un período de dos semestres académicos, se aplicó la misma prueba que se empleó en el pretest, la que suele llamarse prueba de salida o postest para la medición de las dimensiones de la variable y los resultados mostraron una marcada diferencia, en el nivel alto se incrementó 40 puntos con lo cual el $65 \%$ se ubicó en este nivel y en el medio se incrementó 10 puntos, por tanto, el $35 \%$ se ubicó en este nivel. En el nivel bajo no se registró ningún infante (Tabla 3).

Tabla 3. Resultados descriptivos del postest.

\begin{tabular}{lcccc}
\hline & Niveles & Frecuencia & Porcentaje & Porcentaje acumulado \\
\hline Medio & 14 & 35,0 & 35,0 \\
Alto & 26 & 65,0 & 100,0 \\
Total & $\mathbf{4 0}$ & $\mathbf{1 0 0 , 0}$ & \\
\hline
\end{tabular}

En la comparación detallada por grupos de infantes se observó que quienes alcanzaron mayores logros fueron los del grupo de gateadores comprendidos en las edades de seis a 12 meses de edad quienes en el pretest se ubicaron en los niveles medio y bajo y en el postest el 91,7 \% se ubicó en el nivel alto (Tabla 4). Estos resultados son explicables debido a que en este período etario los niños inician la locomoción con el gateo, la bipedestación y los desplazamientos sostenidos en las extremidades inferiores lo cual es el inicio de la construcción de la autonomía en el control músculo esquelético y en los desplazamientos.

Tabla 4. Resultados descriptivos comparativo del pre y postest.

\begin{tabular}{|c|c|c|c|c|c|c|c|c|c|}
\hline & & \multicolumn{2}{|c|}{ Lactantes } & \multicolumn{2}{|c|}{ Gateadores } & \multicolumn{2}{|c|}{ Caminantes } & \multicolumn{2}{|c|}{ Exploradores } \\
\hline & & Recuento & $\%$ de $\mathrm{N}$ & Recuento & $\%$ de $\mathrm{N}$ & Recuento & $\%$ de $\mathrm{N}$ & Recuento & $\%$ de $\mathrm{N}$ \\
\hline \multirow[t]{3}{*}{ Pre } & Bajo & 3 & $50,0 \%$ & 7 & $58,3 \%$ & 12 & $85,7 \%$ & 0 & $0,0 \%$ \\
\hline & Medio & 2 & $33,3 \%$ & 5 & $41,7 \%$ & 1 & $7,1 \%$ & 2 & $25,0 \%$ \\
\hline & Alto & 1 & $16,7 \%$ & 0 & $0,0 \%$ & 1 & $7,1 \%$ & 6 & $75,0 \%$ \\
\hline \multirow[t]{4}{*}{ Post } & Bajo & 0 & $0,0 \%$ & 0 & $0,0 \%$ & 0 & $0,0 \%$ & 0 & $0,0 \%$ \\
\hline & Medio & 3 & $50,0 \%$ & 1 & $8,3 \%$ & 4 & $28,6 \%$ & 6 & $75,0 \%$ \\
\hline & Alto & 3 & $50,0 \%$ & 11 & $91,7 \%$ & 10 & $71,4 \%$ & 2 & $25,0 \%$ \\
\hline & Total & 6 & $100,0 \%$ & 12 & $100,0 \%$ & 14 & $100,0 \%$ & 8 & $100,0 \%$ \\
\hline
\end{tabular}

Nota. Dentro de los lactantes están los infantes con edades hasta los 6 meses; los gateadores (Desde 6 hasta 12 meses); los caminantes (Desde 11 hasta 24 meses); y los exploradores (Desde 24 hasta 36 meses). 
Con la finalidad de aplicar la estadística inferencial, los datos se sometieron a la prueba de normalidad y se encontró que, de los 8 datos, 7 reportaron distribución no normal, por lo que se decidió el uso de estadística no paramétrica, Prueba de rangos con signo Wilcoxon para la prueba de hipótesis.

En la prueba de la hipótesis general en la que se compararon los datos acumulados de la prueba aplicada en el pre y postest, se encontró una significancia $=, 000$, que es menor a, 05 , por lo que, según la regla de decisión se rechazó la H0, debido a que la aplicación del programa influyó de manera significativa en el desarrollo cognitivo de los infantes (Tabla 5).

Tabla 5. Resultados del pre y postest de la variable desarrollo cognitivo.

\begin{tabular}{cc}
\hline & Post_Variable - Pre_Variable \\
\hline $\mathrm{Z}$ & $-5,448^{\mathrm{b}}$ \\
Sig. asintótica (bilateral) &, 000 \\
\hline
\end{tabular}

En la ejecución del procedimiento estadístico para la prueba de la hipótesis específica 1, realizada la comparación de los resultados del pre y postest de la dimensión confianza, se encontró una significancia
$=, 000$, que es menor a, 05 , por lo que, se rechazó la H0, debido a que la aplicación del programa influyó de manera significativa en el desarrollo cognitivo de la confianza (Tabla 6).

Tabla 6. Prueba de significancia de la dimensión confianza.

\begin{tabular}{cc}
\hline & Post_D1_Confianza - Pre_D1_Confianza \\
\hline$Z$ & $-5,174^{\mathrm{b}}$ \\
Sig. asintótica (bilateral) &, 000 \\
\hline
\end{tabular}

La prueba de hipótesis específica 2 realizada con los datos del pre y postest de la dimensión autonomía, arrojó una significancia $=, 000$, que es menor a ,05, por lo que, se rechazó la $\mathrm{H} 0$, debido a que la aplicación del programa influyó de manera significativa en el desarrollo cognitivo de la autonomía (Tabla 7).

Tabla 7. Prueba de significancia de la dimensión autonomía.

\begin{tabular}{cc}
\hline & Post_D2_Autonomía - Pre_D2_Autonomía \\
\hline$Z$ & $-5,249^{\mathrm{b}}$ \\
Sig. asintótica (bilateral) &, 000 \\
\hline
\end{tabular}

Enla prueba dehipótesisespecífica3, comparados los resultados del pre y postest de la dimensión vergüenza/culpa, se encontró una significancia $=$ , 000 , que es menor a ,05, por lo que, se rechazó la
H0, debido a que la aplicación del programa influyó de manera significativa en el desarrollo cognitivo de la vergüenza/culpa (Tabla 8). 
Tabla 8. Prueba de significancia de la dimensión vergüenza/culpa.

\begin{tabular}{cc}
\hline & Post_D3_Vergüenza-culpa Pre_D3_Vergüenza-culpa \\
\hline$Z$ & $-5,380^{\mathrm{b}}$ \\
Sig. asintótica (bilateral) &, 000 \\
\hline
\end{tabular}

\section{Discusión}

Luego de la aplicación del programa de educación temprana, por un período de dos semestres académicos, con cuatro profesores y cinco estudiantes del último ciclo de la carrera de educación inicial a 40 infantes menores de tres años en un establecimiento penitenciario de Lima, se encontró que la educación temprana influye de manera significativa en el desarrollo cognitivo $($ sig $=, 000)$; estos resultados son parecidos a los Dorigo y Janampa (2012) quienes desarrollaron una investigación en Lima-Perú sobre la situación penitenciaria de la mujer gestante y de las madres con hijos privadas de la libertad en un penal de mujeres y concluyeron que no existen servicios de psicología, de pediatría permanente, acceso a talleres y recomendaron que brinde talleres sobre el cuidado de los niños, sugirieron construcción de un espacio acogedor para que las reclusas reciban a sus hijos que las visitan y que se efectúe un mayor esfuerzo para favorecer el vínculo materno filial y familiar a través de programas; sin embargo, los resultados de esta investigación son diferentes a los encontrados por Gasser (2016) quien investigó en Bolivia los procesos de socialización, referentes y modelos sociales en niños cuyos padres viven en las cárceles bolivianas, los resultados mostraron que el encarcelamiento tiene como consecuencia la desesperanza asimilada, especialmente para aquellos que han estado encarcelados durante algunos años, ya que la incertidumbre de su situación legal les impide edificar un proyecto de vida e imaginar un futuro para sí y para sus hijos.
Las diferencias se explican porque la población es de edad diferente y debido a que los niños que participaron del estudio no estaban dentro de la cárcel y quien estaba detenido era el padre y no la madre, como en esta investigación.

La aplicación de un programa de educación temprana influye en el desarrollo cognitivo de la confianza ( $\operatorname{sig}=, 000)$ delos niños; tal como muestran los resultados de esta investigación en los que se evidencia que la relación madre niño en los primeros años debe ser favorecida por los programas debido a los beneficios que aporta, y resultan coherentes con los hallazgos de Montalvo (2007) quien investigó el ejercicio político de la maternidad en la prisión de mujeres del Ecuador, y evidenció que viven su maternidad de manera compartida y colectiva y esta experiencia particular tiene implicaciones para sus hijos; concluyó que se debe hacer un mayor esfuerzo para favorecer el vínculo materno-filial y familiar a través de programas, para evitar la desestructuración familiar y para que el niño crezca sin problemas en su desarrollo. Sin embargo, estos resultados son diferentes a los encontrados por Méndez (2019) quien realizó una investigación en una prisión femenina de Lima, vislumbró los contrastes que existen para convivir con menores, a pesar de compartir las mismas condiciones de encierro, destacó que las redes de soporte dentro y fuera del recinto, son necesarias para llevar a cabo estrategias de cuidados y provisión.

Se encontró que la aplicación de una educación temprana influye en el desarrollo cognitivo de la autonomía $(\operatorname{sig}=, 000)$ de los niños menores de tres 
años, estos resultados muestran que los niños van adquiriendo cierta autonomía en la relación con sus madres lo cual se convierte en un elemento de tranquilidad para ellas en tanto que observan como el infante recurre a conductas de autovalimiento, en ese sentido, estos hallazgos son compatibles con los de Manríquez (2016) quien investigó en Chile, los programas de cuidado de hijos e hijas de mujeres privadas de libertad para registrar y precisar los proyectos y sus particularidades en el cuidado del menor de tres años que se encuentra en centros penitenciarios y concluyó que los niños son un estímulo para las madres que están en prisión y cambian su perspectiva de la vida, queriendo sobresalir y obtener una mejor vida para su familia. Así mismo estos resultados son similares a los Amaya et al. (2008) quienes investigaron las reacciones emocionales, cognitivas y de comportamiento experimentadas por un conjunto de madres privadas de su libertad en San José, Costa Rica y concluyeron que cuando los separan a madres e hijos, quienes han vivido con ellos en el programa Casa Cuna, dan lugar a una serie de recomendaciones y pautas específicas para que el estudio refuerce como las madres deben estar preparadas para la separación de sus hijos para que tengan una buena niñez y logren una buena socialización; las coincidencias se refieren a que tanto la autonomía de los infantes cuanto la preparación de las madres para la separación próxima de sus hijos resultan favorables para ambos y para el sistema.

En esta investigación se encontró que el desarrollo cognitivo de la vergüenza/culpa fue influenciado con la aplicación de una educación temprana (sig $=, 000)$ en los niños menores de tres años, estos resultados son diferentes a los encontrados por Becerra (2004) quien investigó en Colombia sobre las visitas de niños a contexto penitenciario como un espacio para prevenir el maltrato Infantil y fortalecer el vínculo familiar en la que concluyó que los hijos son el motor de vida del interno, la crianza debe ser con base en disciplina con amor; es necesario optimizar en calidad, la cantidad del tiempo compartido en la visita; las diferencias están determinadas por el tipo de población y por el método de estudio. Así mismo, estos resultados difieren de los hallazgos de Merino (2015) quien realizó una investigación en España con la finalidad es conocer la situación del colectivo carcelario, principalmente de las mujeres que tenían niños bajo su cuidado tanto dentro como fuera de la prisión y concluyó que la permanencia de prisión es difícil en las mujeres, es mucho más para las madres, hace imposible desarrollar la maternidad, a pesar de las leyes que hacen creer que es posible; la infraestructura es deficitaria, los módulos adaptados se han reformado, pero no dejan de tener esa esencia de prisión que no permite que las madres sean tales; la diferencia se explica, porque en este estudio las madres permanecían con sus hijos en el interior del centro penitenciario mientras que en el trabajo de Merino hay hijos internos pero también externos.

\section{CONCLUSIONES}

El programa de educación temprana desarrollado con infantes menores de tres años en contextos carcelarios influyó de manera significativa en su desarrollo cognitivo.

La confianza de los infantes menores de tres años en contextos carcelarios mejoró de manera significativa después de la aplicación de un programa de educación temprana.

El desarrollo de la autonomía de los infantes menores de tres años en contextos carcelarios fue favorecido de manera significativa a través de la aplicación de un programa de educación temprana en el que participaron durante un año lectivo. 
La vergüenza como autovaloración de las conductas no aceptadas socialmente fue incorporada de manera básica en infantes menores de tres años en contextos carcelarios a través de la aplicación de un programa de educación temprana en el que participaron durante un año lectivo.

\section{REFERENCIAS}

Aguilera, M. (2011). Mujeres en prisiones españolas. La cárcel del siglo XXI. Desmontando mitos y recreando alternativas, vol. 973. 44 - 48. https://dialnet.unirioja.es/servlet/ articulo? codigo $=3667859$

Amaya, M., Cardona, S., Ramírez, S., Sánchez, L. L. y Gantiva, C. A. (2008). Evaluación de la efectividad de un programa de intervención breve para la disminución de la ansiedad en personas que ingresan por primera vez a un centro penitenciario. Fundación Universitaria Konrad Lorenz. https://www.redalyc.org/ pdf/2972/297224999004.pdf

Antony, C. (2007). Las cárceles femeninas de América Latina. Nueva Sociedad 208, Marzo Abril 2007, ISSN: 0251-3552-

Bordignon, N. A. (2006). El desarrollo psicosocial de Eric Erikson. El diagrama epigenético del adulto. Corporación Universitaria Lasallista Colombia. https://www.redalyc.org/pdf/695/69520210.pdf

Becerra, L. (2004). La visita de niños y niñas en contexto penitenciario y Carcelario, un espacio para prevenir el maltrato Infantil y fortalecer el vínculo familiar. (Tesis de grado). Pontificia Universidad Javeriana de Bogotá.

Briceño, M. (2006). Mujeres y prisión en Colombia: Análisis desde una perspectiva de Derechos humanos y género. Procuraduría Delegada en lo Preventivo para Derechos Humanos y Asuntos Étnicos, Grupo de Asuntos Penitenciarios y Carcelarios. Apoyo técnico y financiero del Fondo de Desarrollo de las Naciones Unidas para la Mujer. Bogotá: Unifem

Boeree, G. (2002). Teoría de la personalidad de Erik Erikson. Revista Lasallista de investigación - vol. 2 no. 2
Bowlby, J. (1988). Una base segura. Aplicaciones clínicas de una teoría del apego. Paidós. https://www.bibliopsi.org/docs/carreras/ obligatorias/CFG/11ninez/cleici/primer\%20 cuatrimestre\%202020/UNA_BASE_ SEGURA-_LA_TEORIA_DEL_APEGO.pdf

Chokler, M. (1988). Los Organizadores del Desarrollo Psicomotor. Ediciones Cinco. https://es.slideshare.net/cindycopello/losorganizadores-del-desarrollo-psicomotorlibro-completo

Dorigo, N. y Janampa, M. (2012). Detrás del muro hay madres Diagnostico situacional: Brecha entre la normativa y la situación penitenciaria de la mujer gestante y madres con hijos privada de la libertad en el penal de mujeres de Chorrillos I. Pontificia Universidad Católica del Perú. https:// sumaqmusquy.files.wordpress.com/2013/06/ detras-del-muro-hay-madres-4-12-12.pdf

Erikson, E. O. (1998). Ciclo de vida completo. Artmed. Educación social 47. 129-132

Gasser, P. (2016). Procesos de socialización, referente s y modelos sociales en niños que viven en las cárceles bolivianas. Universidad Complutense de Madrid. https://eprints.ucm.es/id/ eprint/37653/1/T37212.pdf

Instituto Nacional de las Mujeres (2002). Niños $y$ mujeres invisibles. Hijas e hijos de reclusas. Fondo de las naciones Unidas para la Infancia. http://cedoc.inmujeres.gob.mx/documentos_ download/100836.pdf

Instituto Nacional Penitenciario (2012). Plan Estratégico Institucional Periodo 2012 -2016. Lima: "Informe sobre las mujeres privadas de libertad", 2006 Extraído el 25 de abril de 2019 de: http://www.artemisanoticias.com.ar/images/ FotosNotas/Informe\%20sobre\%20mujeres\%20 privadas\%20de\%20libertad.pdf

Kalinsky, B. (2013). Epistemología del filicidio: violencia contra las mujeres. VOX JURIS, Lima (Perú) 26(2): 215-236,2013

Manríquez, M. (2016). Revisión sobre desarrollo emocional $y$ apego seguro de hijos e hijas de madres privadas de libertad en contexto de programas de residencias transitorias en 
recintos penitenciarios, una mirada desde la salud pública. Chile: Universidad de Chile. http://bibliodigital.saludpublica.uchile.cl:8080/ dspace/bitstream/handle/123456789/510/ Tesis_Monica\% $20 \mathrm{Manr} \% \mathrm{C} 3 \% \mathrm{ADquez}$. pdf? sequence $=1$ \&isAllowed $=y$

Méndez, A. P. (2019). "Cuidados encerrados" Organización social del cuidado infantil en una prisión femenina de Lima. Pontificia Universidad católica del Perú. http:// tesis.pucp.edu.pe/repositorio/bitstream/ handle/20.500.12404/15545/MENDEZ_ ANA_CUIDADOS_ENCERRADOS. pdf? sequence $=1 \&$ is Allowed $=y$

Merino, S. (2015). Madres entre rejas, hijos condenados. Universidad de Valladolid. http:// uvadoc.uva.es/handle/10324/15984

Montalvo, M. A. (2007). El ejercicio político de la maternidad en la cárcel de mujeres de Quito: testimonios y perspectivas. Universidad Andina Simón Bolívar. https://1library.co/document/ yjkxw96q-ejercicio-politico-maternidad-carcelmujeres-quito-testimonios-perspectivas.html

Moreno, M. T. (2012). Interacciones vinculares en el sistema de cuidado infantil en contextos depobreza $y$ desnutrición crónica temprana. Centro de
Estudios Avanzados en Niñez y Juventud alianza de la Universidad de Manizales y el CINDE. http://bibliotecavirtual.clacso.org.ar/Colombia/ alianza-cinde-umz/20130402125354/Te sisMariaTeresaMorenoZavaleta.pdf

Ramírez-Abrahams, P., Patiño-Mora, V. y GamboaVasquez, E. (2014). Early Education from Birth to Age 3: Three Analylitical Perspectives. Educare Electronic Journal ISSN: 1409-4258 Vol. 18(3). 67-90

Reglamento del Código de Ejecución Penal. (2003). Artículo 12

Robertson, O. (2012). Convictos colaterales: niños $y$ niñas de progenitores presos. Quaker United Nations Office. https://www.quno.org/sites/ default/files/resources/ESPAN\%CC\%83OL_ Collateral\%20Convicts_Recommendations $\% 20$ and $\% 20$ good\%20practice.pdf

Tamayo y Tamayo, M. (2003). El proceso de la Investigación Científica. Limusa. ISBN 968-185872-7

Wallon, H. (1976). Le développement psychiomotor l'enfante. Enfance 75(3) pp. 37-55 